\title{
Specificity of the Linear Array HPV Genotyping Test for detecting human papillomavirus genotype 52 (HPV-52)
}

\author{
Anja Oštrbenk ${ }^{1}$, Boštjan J. Kocjan ${ }^{1}$, Mario Poljak ${ }^{1 凶}$
}

\begin{abstract}
Introduction: HPV-52 is one of the most frequent human papillomavirus (HPV) genotypes causing significant cervical pathology. The most widely used HPV genotyping assay, the Roche Linear Array HPV Genotyping Test (Linear Array), is unable to identify HPV52 status in samples containing HPV-33, HPV-35, and/or HPV-58.

Methods: Linear Array HPV-52 analytical specificity was established by testing 100 specimens reactive with the Linear Array HPV33/35/52/58 cross-reactive probe, but not with the HPV-33, HPV-35, and/or HPV-58 individual probes, using an HPV-52-specific real-time PCR assay with a detection level of 3.9 DNA copies/reaction. In addition, we established the prevalence of HPV-52 in 49 samples reactive with the Linear Array HPV-33/35/52/58 cross-reactive probe, but containing either HPV-33, HPV-35, and/or HPV58 using the same approach.

Results: The HPV-52-specific assay detected HPV-52 in all 100 samples reactive with the Linear Array HPV-33/35/52/58 cross-reactive probe, but not with the HPV-33, HPV-35, and/or HPV-58 probes. The presence of up to six other HPV genotypes did not influence the HPV-52 analytical performance of Linear Array. Only 6/49 (12.2\%) samples reactive with the Linear Array HPV-33/35/52/58 cross-reactive probe, but containing either HPV-33, HPV-35 or/and HPV-58, were HPV-52 positive.

Conclusions: Linear Array is a highly reliable test for detecting HPV-52 in the absence of genotypes HPV-33, HPV-35, and HPV-58. Additional testing using an HPV-52-specific test is necessary when HPV-33, HPV-35, and/or HPV-58 are present in the sample.
\end{abstract}

Keywords: HPV, human papillomaviruses, Linear Array, HPV genotype 52, HPV-52

Received: 29 July 2014 | Returned for modification: 15 August 2014 | Accepted: 29 August 2014

\section{Introduction}

Cervical cancer affects 530,000 women annually and is still one of the three leading cancers in $90 \%$ of countries worldwide $(1,2)$. It has been widely demonstrated that persistent infection with human papillomaviruses (HPV) is etiologically linked with cervical cancer (2). In 2009, the International Agency for Research on Cancer (IARC) classified 12 HPV genotypes (HPV-16, HPV-18, HPV-31, HPV-33, HPV-35, HPV-39, HPV-45, HPV-51, HPV-52, HPV-56, HPV58, and HPV-59) as high-risk HPV (hrHPV) genotypes (3). HPV-16 is the major causative agent of cervical cancer worldwide and also the HPV genotype most commonly found in women with normal cervical cytological findings, followed by HPV-18, HPV-52, HPV-31, and HPV-58 (4).

HPV-52 is one of the most frequent hrHPV genotypes causing significant cervical pathology. It was first characterized by Wayne Lancaster in May 1987 (5) and is classified taxonomically in the genus Alphapapillomavirus (Alpha-PV) as species Alpha-9. In a recent meta-analysis on HPV genotype distribution across the complete spectrum of cervical disease, HPV-52 ranked second in women with a cytological finding of atypical squamous cells of undetermined significance, third in women with high-grade squamous intraepithelial lesions, and third in women with histologically confirmed cervical intraepithelial neoplasia grade 3 (CIN3) (6). However, the relative etiological importance of HPV-52 significantly dropped between $\mathrm{CIN}_{3}$ and invasive cervical cancers (cancer:CIN3 ratio 0.35), a pattern that was also seen for all other hrHPV genotypes except HPV-16, HPV-18, and HPV-45 (6). Similarly, in Slovenia HPV-52 is the fourth most frequently identified hrHPV genotype in women with normal cytology (7), sixth among women with $\mathrm{CIN}_{3}$ (8), but ranked only tenth in women with inva- sive cervical cancer (9).

In a recent review of commercially available HPV tests, at least 125 distinct tests for detecting Alpha-PVs were identified with at least 84 variants, but unfortunately no single publication in peerreviewed literature could be found for more than $75 \%$ of HPV tests (10). HPV DNA full genotyping tests, which allow individual determination of several Alpha-PV genotypes, including all 12 officially recognized hrHPV genotypes, are the largest group of currently available commercial HPV tests. Currently, these tests are indispensable as research tools, but their clinical value has not been finally determined (10). As the use of prophylactic HPV vaccines becomes more widespread, surveillance for populationlevels of effectiveness is becoming an increasingly important activity, which requires the use of standardized HPV genotyping tests with analytical performance different from tests with clinically validated cutoffs; for example, tests with higher analytical sensitivity and absolute analytical specificity (10).

The currently most frequently used HPV full genotyping tests utilize the principle of reverse hybridization. In these tests, a fragment of the HPV genome is first PCR-amplified with biotinlabeled primers, and the resulting amplicons are then denatured and detected using HPV genotype-specific probes immobilized on a strip, filter (DNA microarray chip), or microtiter well. The Linear Array HPV Genotyping Test (Linear Array; Roche Molecular Diagnostics, Pleasanton, CA) currently has the most abundant data in peer-reviewed literature among commercially available genotyping tests (10). Linear Array combines PCR-amplification and reverse-line blot hybridization for simultaneously detecting 36 individual HPV genotypes (HPV-6, HPV-11, HPV-16, HPV-18, HPV-26, HPV-31, HPV-33, HPV-35, HPV-39, HPV-40, HPV-42, HPV-44, HPV45, HPV-51, HPV-52, HPV-53, HPV-54, HPV-56, HPV-58, HPV-59, 
HPV-61, HPV-62, HPV-64, HPV-66, HPV-67, HPV-68, HPV-69, HPV70, HPV-71, HPV-72, HPV-73, HPV-81, HPV-82, HPV-83, HPV-84, and HPV-89), and one HPV subtype (subHPV-82 or IS39), including all $12 \mathrm{hrHPV}$ genotypes. Additional primer pairs targeting the human $\beta$-globin gene provide a control for cell adequacy, extraction, and amplification. Because directly detecting HPV-52 in Linear Array is prohibited due to intellectual property rights, HPV-52 is identified using a probe that cross-reacts with HPV-33, HPV-35, and HPV-58 (11-13). Therefore Linear Array is unable to correctly determine HPV-52 status in women co-infected with either HPV-33, HPV-35, and/or HPV-58 (10, 11). Because the manufacturer of Linear Array does not provide any supplementary test to resolve the final HPV52 status in specimens containing HPV-33, HPV-35, and/or HPV-58, correctly detecting HPV-52 DNA in these samples currently relies merely on different in-house HPV-52-specific assays (11-14), including Universal ProbeLibrary-based HPV 52-specific real-time PCR, developed in our laboratory (12).

In the first part of this study, the analytical specificity of Linear Array for detecting HPV-52 was established by testing 100 routine specimens reactive with the Linear Array HPV-33/35/52/58 crossreactive probe, but not with the HPV-33, HPV-35, and/or HPV-58 genotype-specific individual probes, using a rapid and reproducible HPV-52-specific real-time PCR assay with a detection level of 3.9 DNA copies/reaction (12). Using the same approach, in the second part of the study we established the prevalence of HPV-52 infection in samples reactive with the Linear Array HPV-33/35/52/58 cross-reactive probe, but also containing either HPV-33, HPV-35, and/or HPV-58.

\section{Methods}

For the first part of the study, 100 consecutive routine cervical smears obtained from the same number of Slovenian women, which tested reactive with the Linear Array HPV-33/35/52/58 crossreactive probe, but not with the individual HPV-33, HPV-35, and/or HPV-58 genotype-specific probes (defined for the purpose of this study as "Linear Array true HPV-52-positive") were selected from cervical smear archives at the Institute of Microbiology and Immunology, Faculty of Medicine, University of Ljubljana, Slovenia (Table 1). For the second part of the study, 49 consecutive samples defined for the purpose of this study as "Linear Array possible HPV-52-positive" - samples which reacted positively with an HPV33/35/52/58 cross-reactive hybridization probe and in addition also with probe(s) specific for either HPV-33 (eight samples), HPV-35 (12 samples), and/or HPV-58 (31 samples) were selected from the same archives (Table 2).

All samples included in the study were tested initially for the presence of 13 and $14 \mathrm{hrHPV}$ with either of two clinically validated HPV assays: the Hybrid Capture 2 HPV DNA Test (Qiagen, Hilden, Germany) and/or the RealTime High Risk HPV test (Abbott, Wiesbaden, Germany), respectively, according to the manufacturer's instructions and as previously described (15). Linear Array was performed according to the manufacturer's instructions (11).

An HPV-52-specific real-time PCR assay with a detection level of 3.9 HPV-52 DNA copies/reaction was performed as previously described (12). Briefly, the assay was performed on a LightCycler 1.5 Real-time PCR instrument (Roche Diagnostics) in a $20 \mu \mathrm{l}$ reaction mixture containing water, $5 \mu$ l of template DNA, $5 \mu$ l of $5 \times$ LightCycler TaqMan Master Mix (Roche Diagnostics), $0.8 \mu \mathrm{M}$ of each of the primers, and $0.2 \mu \mathrm{M}$ of TaqMan probe (12).

\section{Results}

As shown in Table 1, among 100 samples used to evaluate Linear Array HPV-52 analytical specificity, 34 samples contained HPV-52 only, 21 samples contained one additional HPV genotype, 18 samples contained two additional HPV genotypes, and 27 samples contained three to six additional HPV genotypes. A clearly detectable fluorescence signal using an HPV-52-specific real-time PCR assay was obtained in all 100 "Linear Array true HPV-52-positive" samples, resulting in $100 \%$ analytical specificity of the Linear Array for detecting HPV-52. The presence of several other HPV genotypes (for details, see Table 1) did not influence the analytical performance of either Linear Array or an HPV-52-specific real-time PCR assay for detecting HPV-52.

Of the 49 samples labelled as "Linear Array possible HPV-52positive", only six samples tested positive with an HPV-52-specific real-time PCR assay (samples 144-149, Table 2), giving $12.2 \%$ prevalence of HPV-52 infection in samples reactive with the Linear Array HPV-33/35/52/58 cross-reactive probe, but containing HPV33 and/or HPV-35 and/or HPV-58. As shown in Table 2, among 43 "Linear Array possible HPV-52-positive" samples that tested HPV52-negative using an HPV-52-specific real-time PCR assay (samples 101-143, Table 2), 26 (60.5\%) samples contained HPV-58, 11 (25.6\%) samples contained HPV-35, five (11.6\%) samples contained HPV-33, and one sample (2.3\%) contained HPV-33 and HPV-58 (sample 132, Table 2). Among six "Linear Array possible HPV-52positive" samples that tested HPV-52-positive using an HPV-52specific real-time PCR assay (samples 144-149, Table 2), three samples contained HPV-58, one sample contained HPV-35, one sample contained HPV-33, and one sample contained HPV-33 and HPV-58.

\section{Discussion}

In the past two decades, various tests for detecting and genotyping a broad spectrum of HPV genotypes have been developed, validated, and introduced into clinical laboratories (10). In addition to recently introduced HPV tests that can discriminate HPV-16 and HPV-18 (both account for $73 \%$ of all cervical cancer worldwide) from other hrHPV genotypes (15-17), there is increased interest in sensitive and specific genotyping tests that will be necessary to evaluate real-life clinical efficacy of prophylactic HPV vaccines and will impact current and future HPV vaccination programs (18).

In this study, we evaluated the analytical specificity of Linear Array, the most commonly used HPV genotyping assay $(10,18)$, to detect HPV-52 in the absence/presence of genotypes HPV-33, HPV-35, and HPV-58. HPV-52 is a hrHPV genotype that is highly prevalent in various Asian and African countries and accounts for a larger fraction of cervical cancers in these regions (6). Knowledge of the exact prevalence and geographical distribution of HPV-52 is thus important for designing and implementing next-generation HPV vaccines with a broader spectrum of protection than current HPV vaccines and for designing the next generation of clinically useful HPV tests. Due to the inability of Linear Array to resolve the HPV-52 status in specimens containing HPV-33, HPV-35, and/or HPV-58, correctly detecting HPV-52 DNA in these samples mainly relies on in-house HPV-52-specific assays. Several different inhouse HPV-52-specific real-time PCR-based assays have been developed in the past years $(11-14,19)$. Sensitivity and specificity of HPV-52-specific real-time PCR developed by Coutlee et al. in 2007 were evaluated on 265 anogenital samples obtained from 
Table 1 | Distribution of HPV genotypes in 100 samples that tested positive with the HPV-33/35/52/58 cross-reactive probe, but not with the HPV-33, HPV-35, or HPV-58 genotype-specific individual probes with HPV Linear Array (“Linear Array true HPV-52-positive" samples), assessed by using an HPV52-specific real-time PCR assay.

\begin{tabular}{|c|c|c|c|}
\hline Sample no. & Linear Array result & $\begin{array}{l}\text { HPV-52 real-time } \\
\text { PCR assay result }\end{array}$ & Frequency \\
\hline $1-34$ & 52 & Positive & 34 \\
\hline $35-38$ & 16,52 & Positive & 4 \\
\hline 39,40 & 18,52 & Positive & 2 \\
\hline 41,42 & 31,52 & Positive & 2 \\
\hline 43 & 42,52 & Positive & 1 \\
\hline 44 & 45,52 & Positive & 1 \\
\hline 45 & 52,54 & Positive & 1 \\
\hline 46 & 52,56 & Positive & 1 \\
\hline 47 & 52,59 & Positive & 1 \\
\hline 48 & 52,61 & Positive & 1 \\
\hline 49,50 & 52,62 & Positive & 2 \\
\hline $51-53$ & 52,66 & Positive & 3 \\
\hline 54 & 52,83 & Positive & 1 \\
\hline 55 & 52,89 & Positive & 1 \\
\hline 56 & $16,42,52$ & Positive & 1 \\
\hline 57 & $16,52,59$ & Positive & 1 \\
\hline 58 & $16,52,89$ & Positive & 1 \\
\hline 59 & $18,39,52$ & Positive & 1 \\
\hline 60 & $18,52,59$ & Positive & 1 \\
\hline 61 & $18,52,82$ & Positive & 1 \\
\hline 62 & $31,45,52$ & Positive & 1 \\
\hline 63 & $31, \mathbf{5 2}, 54$ & Positive & 1 \\
\hline 64 & $39,51,52$ & Positive & 1 \\
\hline 65 & $39, \mathbf{5 2}, 54$ & Positive & 1 \\
\hline 66 & $42, \mathbf{5 2}, 56$ & Positive & 1 \\
\hline 67 & $42,52,66$ & Positive & 1 \\
\hline 68 & $45,52,89$ & Positive & 1 \\
\hline 69 & $51, \mathbf{5 2}, 58$ & Positive & 1 \\
\hline 70 & $52,59,73$ & Positive & 1 \\
\hline 71 & $52,61,73$ & Positive & 1 \\
\hline 72 & $52,62,89$ & Positive & 1 \\
\hline 73 & $52,66,89$ & Positive & 1 \\
\hline 74 & $6,16,52,66$ & Positive & 1 \\
\hline 75 & $6,39,52,67$ & Positive & 1 \\
\hline 76 & $16,18,35,52$ & Positive & 1 \\
\hline 77 & $16,42,52,83$ & Positive & 1 \\
\hline 78 & $16,45,51,52$ & Positive & 1 \\
\hline 79 & $16,52,59,66$ & Positive & 1 \\
\hline 80 & $16,52,61,84$ & Positive & 1 \\
\hline 81 & $18,51,52,53$ & Positive & 1 \\
\hline 82 & $18,52,56,62$ & Positive & 1 \\
\hline 83 & $18,52,66,84$ & Positive & 1 \\
\hline 84 & $18, \mathbf{5 2}, 73,89$ & Positive & 1 \\
\hline 85 & $31,52,53,70$ & Positive & 1 \\
\hline 86 & $39,42,52,55$ & Positive & 1 \\
\hline 87 & $39,42,52,83$ & Positive & 1 \\
\hline 88 & $42,52,56,68$ & Positive & 1 \\
\hline 89 & $42,52,58,59$ & Positive & 1 \\
\hline 90 & $45,52,58,62$ & Positive & 1 \\
\hline 91 & $52,53,54,62$ & Positive & 1 \\
\hline 92 & $52,53,62,89$ & Positive & 1 \\
\hline 93 & $16,51,52,59,82$ & Positive & 1 \\
\hline 94 & $31,39,45,52,66$ & Positive & 1 \\
\hline 95 & $51,52,61,82,84$ & Positive & 1 \\
\hline 96 & $52,53,59,73,84$ & Positive & 1 \\
\hline 97 & $16,52,53,59,66,84$ & Positive & 1 \\
\hline 98 & $16,52,54,61,66,73$ & Positive & 1 \\
\hline 99 & $39,45,52,53,61,62,68$ & Positive & 1 \\
\hline 100 & $6,11,40,51,52,59,84$ & Positive & 1 \\
\hline
\end{tabular}

Canadian women, and tests showed excellent agreement with conventional HPV-52 typing methods (14). Stevens et al. developed the Roche Light Cycler 480-based HPV-52 highly specific real-time PCR assay in 2008, with sensitivity equivalent to Linear Array, which was successfully evaluated on a panel of Australian samples con-
Table 2 | Distribution of HPV genotypes in 49 samples that tested positive with the HPV-33/35/52/58 cross-reactive probe and with at least one of the HPV genotype-specific probes for HPV-33, HPV-35, or HPV-58 with HPV Linear Array ("Linear Array possible HPV-52-positive" samples), assessed by using an HPV-52-specific real-time PCR assay.

\begin{tabular}{|c|c|c|c|c|}
\hline Sample & $\begin{array}{l}\text { Linear Array } \\
\text { result }\end{array}$ & $\begin{array}{l}\text { HPV-52-specific } \\
\text { real-time } \\
\text { PCR assay } \\
\text { results }\end{array}$ & $\begin{array}{c}\text { HPV } \\
\text { genotype(s) }\end{array}$ & Frequency \\
\hline 101,102 & $33,52 *$ & Negative & 33 & 2 \\
\hline $103-105$ & $35,52 *$ & Negative & 35 & 3 \\
\hline $106-114$ & $52^{\star}, 58$ & Negative & 58 & 9 \\
\hline 115 & $16,35,52^{*}$ & Negative & 16,35 & 1 \\
\hline $116-118$ & $16,52^{\star}, 58$ & Negative & 16,58 & 3 \\
\hline 119 & $18,33,52^{*}$ & Negative & 18,33 & 1 \\
\hline 120 & $18,35,52^{*}$ & Negative & 18,35 & 1 \\
\hline 121 & $31,35,52^{*}$ & Negative & 31,35 & 1 \\
\hline 122 & $35,51,52^{*}$ & Negative & 35,51 & 1 \\
\hline 123 & $35,52 *, 89$ & Negative & 35,89 & 1 \\
\hline 124 & $52 *, 53,58$ & Negative & 53,58 & 1 \\
\hline 125 & $52 *, 58,59$ & Negative & 58,59 & 1 \\
\hline 126 & $52 *, 58,66$ & Negative & 58,66 & 1 \\
\hline 127,128 & $52 *, 58,84$ & Negative & 58,84 & 2 \\
\hline 129 & $6,51,52^{*}, 58$ & Negative & $6,51,58$ & 1 \\
\hline 130 & $16,35,52 *, 59$ & Negative & $16,35,59$ & 1 \\
\hline 131 & $16,35,52^{*}, 89$ & Negative & $16,35,89$ & 1 \\
\hline 132 & $33,42,52 *, 58$ & Negative & $33,42,58$ & 1 \\
\hline 133 & $52 *, 53,56,58$ & Negative & $53,56,58$ & 1 \\
\hline 134 & $52^{*}, 58,82,89$ & Negative & $58,82,89$ & 1 \\
\hline 135 & $\begin{array}{c}16,51,52^{*} \\
58,84\end{array}$ & Negative & $\begin{array}{c}16,51 \\
58,84\end{array}$ & 1 \\
\hline 136 & $\begin{array}{c}16,52^{*}, 53 \\
58,61\end{array}$ & Negative & $\begin{array}{c}16,53 \\
58,61\end{array}$ & 1 \\
\hline 137,138 & $\begin{array}{c}51,52^{*}, 58 \\
\quad 61,83\end{array}$ & Negative & $\begin{array}{l}51,58 \\
61,83\end{array}$ & 2 \\
\hline 139 & $\begin{array}{c}52^{*}, 53,58 \\
66,89\end{array}$ & Negative & $\begin{array}{l}53,58 \\
66,89\end{array}$ & 1 \\
\hline 140 & $\begin{array}{c}16,18,33,39 \\
52^{*}, 68\end{array}$ & Negative & $\begin{array}{c}16,18,33 \\
39,68\end{array}$ & 1 \\
\hline 141 & $\begin{array}{c}31,45,51,52 * \\
53,58\end{array}$ & Negative & $\begin{array}{c}31,45,51 \\
53,58\end{array}$ & 1 \\
\hline 142 & $\begin{array}{c}16,35,51,52^{*} \\
53,55,56,84\end{array}$ & Negative & $\begin{array}{c}16,35,51 \\
53,55,56 \\
84\end{array}$ & 1 \\
\hline 143 & $\begin{array}{c}16,18,31,33 \\
51,52^{*}, 53,84 \\
89\end{array}$ & Negative & $\begin{array}{c}16,18,31 \\
33,51,53 \\
84,89\end{array}$ & 1 \\
\hline 144 & $33,52^{*}$ & Positive & 33,52 & 1 \\
\hline 145 & $33,52 *, 58$ & Positive & $33,52,58$ & 1 \\
\hline 146 & $42,51,52^{\star}, 58$ & Positive & $\begin{array}{l}42,51 \\
52,58\end{array}$ & 1 \\
\hline 147 & $\begin{array}{c}52 *, 54,58 \\
81,89\end{array}$ & Positive & $\begin{array}{c}52,54,58 \\
81,89\end{array}$ & 1 \\
\hline 148 & $\begin{array}{c}35,52 *, 62 \\
83,84\end{array}$ & Positive & $\begin{array}{c}35,52,62 \\
83,84\end{array}$ & 1 \\
\hline 149 & $\begin{array}{c}51,52 *, 53,56 \\
58,61,62,84\end{array}$ & Positive & $\begin{array}{c}51,52,53 \\
56,58,61 \\
62,84\end{array}$ & 1 \\
\hline
\end{tabular}

${ }^{\star}$ Co-infection with HPV-52 cannot be ruled out.

taining both single and multiple HPV genotypes (13). In the following years, two additional HPV-52-specific real-time PCRs were developed based on TaqMan chemistry $(11,12)$. More recently, a new HPV-52-specific real-time TaqMan-based assay targeting the E6/E7 region was developed, which can be used as a duplex assay (simultaneously detecting beta-globin) or as a single-target detection assay (19).

For determining HPV-52 status in this study, we used an HPV52-specific real-time PCR assay with a detection level of 3.9 DNA 
copies/reaction developed in our laboratory in 2010 (12). In the initial evaluation, the test proved to be highly specific, sensitive, and reproducible for detecting HPV-52 infection in clinical samples and confirming the presence/absence of HPV-52 in clinical samples tested Linear Array-positive for HPV-52 in the presence of HPV-33, HPV-35, and/or HPV-58 (12). Because our test targets the E6 gene of HPV-52, which is always retained after potential viral integration into the host-cell chromosome and is actively expressed in cervical carcinoma, it could significantly diminish the problems of detecting integrated forms of HPV-52 that may lack larger parts of the viral genome.

In this study, using an HPV-52-specific in-house real-time PCR assay we further confirmed the exceptional analytical specificity of Linear Array for detecting HPV-52. The presence of several other

\section{References}

1. Bosch FX. The path to eliminate cervical cancer in the world and the challenges of professional education. Vaccine. 2012;30:xi-xii.

2. Forman D, de Martel C, Lacey CJ, Soerjomataram I, Lortet-Tieulent J, Bruni L, et al. Global burden of human papillomavirus and related diseases. Vaccine. 2012;30:F12-F23.

3. Bouvard V, Baan R, Straif K, Grosse Y, Secretan B, El Ghissassi F, et al. A review of human carcinogens-part B: biological agents. Lancet Oncol. 2009;10:321-2.

4. Bruni L, Diaz M, Castellsague X, Ferrer E, Bosch FX, de Sanjose S. Cervical human papillomavirus prevalence in 5 continents: meta-analysis of 1 million women with normal cytological findings. J Infect Dis. 2010;202:1789-99.

5. Delius H, Hofmann B. Primer-directed sequencing of human papillomavirus types. Curr Top Microbiol Immunol. 1994;186:13-31.

6. Guan P, Howell-Jones R, Li N, Bruni L, de Sanjose S, Franceschi S, et al. Human papillomavirus types in 115,789 HPV-positive women: A meta-analysis from cervical infection to cancer. Int J Cancer. 2012;131:2349-59.

7. Učakar V, Poljak M, Klavs I. Pre-vaccination prevalence and distribution of highrisk human papillomavirus (HPV) types in Slovenian women: a cervical cancer screening based study. Vaccine. 2012;30:116-20.

8. Kovanda A, Juvan U, Šterbenc A, Kocjan BJ, Seme K, Jančar N, et al. Pre-vaccination distribution of human papillomavirus (HPV) genotypes in women with cervical intraepithelial neoplasia grade 3 ( $\left.\mathrm{CIN}_{3}\right)$ lesions in Slovenia. Acta Dermatovenerol Alp Panonica Adriat. 2009;18:47-52.

9. Jančar N, Kocjan BJ, Poljak M, Lunar MM, Vrtačnik Bokal E. Distribution of human papillomavirus genotypes in women with cervical cancer in Slovenia. Eur J Obstet Gynecol Reprod Biol. 2009;145:184-8.

10. Poljak M, Cuzick J, Kocjan BJ, Iftner T, Dillner J, Arbyn M. Nucleic acid tests for the detection of alpha human papillomaviruses. Vaccine. 2012;30:F100-6.

11. Marks M, Gupta SB, Liaw KL, Kim E, Tadesse A, Coutlee F, et al. Confirmation and quantitation of human papillomavirus type 52 by Roche Linear Array using HPV 52-specific TaqMan E6/E7 quantitative real-time PCR. J Virol Methods. 2009;156:152-6.
HPV genotypes (up to seven different HPV genotypes) did not influence the analytical performance of Linear Array. Based on our results, the confirmation of HPV-52 status in samples reactive with the Linear Array HPV-33/35/52/58 cross-reactive probe, but not with the HPV-33, HPV-35, and/or HPV-58 genotype-specific individual probes ("Linear Array true HPV-52-positive"), using supplementary test(s) is not necessary and is not recommended. In contrast, the second part of this study showed a relatively low prevalence of HPV-52 infection in samples reactive with the Linear Array HPV33/35/52/58 cross-reactive probe, but containing HPV-33, and/or HPV-35 and/or HPV-58 ("Linear Array possible HPV-52-positive" samples). In such samples, however, the confirmation of HPV-52 status using HPV-52-specific tests, such as HPV-52 real-time PCRs, is obligatory and highly recommended.

12. Kocjan BJ, Poljak M, Seme K. Universal ProbeLibrary based real-time PCR assay for detection and confirmation of human papillomavirus genotype 52 infections. J Virol Methods. 2010;163:492-4.

13. Stevens MP, Garland SM, Tabrizi SN. Development and validation of a real-time PCR assay specifically detecting human papillomavirus 52 using the Roche LightCycler 480 system. J Virol Methods. 2008;147:290-6.

14. Coutlee F, Rouleau D, Ghattas G, Hankins C, Vezina S, Cote P, et al. Confirmatory real-time PCR assay for human papillomavirus (HPV) type 52 infection in anogenital specimens screened for HPV infection with the Linear Array HPV genotyping test. J Clin Microbiol. 2007;45:3821-3.

15. Poljak M, Oštrbenk A, Seme K, Učakar V, Hillemanns P, Vrtačnik Bokal E, et al. Comparison of clinical and analytical performance of the Abbott Realtime High Risk HPV test to the performance of hybrid capture 2 in population-based cervical cancer screening. J Clin Microbiol. 2011;49:1721-9.

16. Huang S, Erickson B, Tang N, Mak WB, Salituro J, Robinson J, et al. Clinical performance of Abbott RealTime High Risk HPV test for detection of high-grade cervical intraepithelial neoplasia in women with abnormal cytology. J Clin Virol. 2009;45(Suppl 1):S19-23.

17. Lapierre SG, Sauthier P, Mayrand MH, Dufresne S, Petignat P, Provencher D, et al. Human papillomavirus (HPV) DNA triage of women with atypical squamous cells of undetermined significance with cobas 4800 HPV and Hybrid Capture 2 tests for detection of high-grade lesions of the uterine cervix. J Clin Microbiol. 2012;50:1240-4.

18. Eklund C, Forslund O, Wallin KL, Zhou T, Dillner J. The 2010 global proficiency study of human papillomavirus genotyping in vaccinology. J Clin Microbiol. 2012;50:2289-98.

19. Onyekwuluje JM, Steinau M, Swan DC, Unger ER. A real-time PCR assay for HPV 52 detection and viral load quantification. Clin Lab. 2012;58:61-6. 